MAGNETIC FIELD-CHROMOSPHERIC PLASMA INTERACTION AND THE PROBLEM OF THE BRAKING FORCE IN SURGE DYNAMICS

\title{
V. Dermendjiev
}

Department of Astronomy and NAO

72 Lenin Blvd.

1784 Sofia

Bulgaria

\section{INTRODUCTION}

An important stage in the evolution of solar flares is the generation of a number of dynamic phenomena, greatly varying in size and velocity, but actually representing plasma ejections. They are responsible for the transport of mass and energy and are usually considered to be result of the development of local magnetic features accompanied by a magnetic energy increase in the solar atmosphere. One of these phenomena is the surge prominence. In this contribution, we propose a possible explanation for the nature of the braking force acting in the decelerated ascending phase of surge dynamics. The possibility of structuralization of the chromospheric plasma during surge formation and the plasma action on the external, colimating magnetic field is taken into account.

\section{OBSERVATIONAL AND THEORETICAL CONSIDERATIONS}

This type of prominences is described in [1] as a colimate stream of chromospheric plasma. In [2], ballistical maintenance by dynamic effects was proposed. Some observations [3] suggest that the surge is a cluster of a large number of fine jets with a diameter l", apparently connected with the bright knots, or Ellerman bombs in the lower chromosphere. Likewise, rotational motions in kernel structures of the surges are observed. On the other hand the behaviour of Ellerman bombs in $\mathrm{H}$-alpha [4] suggests their association with bipolar emerging magnetic flux. In [5] it was indicated that the nonlinear effects during smallscale magnetic field penetration in the lower chromosphere lead to temperature and density increase, sufficient to create a gas dynamic driving force for a surge-like jet.

One of the peculiarities in surge dynamics is the so-called braking or retarding effect. It means (see for example [3]) that the deceleration of the outward-moving plasma which takes place after the maximum velocity has been reached is stronger than when gravity alone is acting.

Using the above mentioned observational and theoretical results, we make an attempt at constructing a model of the surge phenomena 
explaining the braking effect in surge dynamics.

\section{THE NATURE OF THE BRAKING FORCE}

We adopt a surge model which represents the phenomenon as a cluster of surge-like jets, each generated by a temperature gradient on the top of a chromospheric bright point. Some hydrodynamical considerations (see for example [6]) lead to the idea that each surge-like jet could be treated as a buoyant vortex structure starting as a forced plume. The velocity profile

$$
\dot{z}(\tau)=\Gamma(4 \pi R)^{-1}\left[\ln \left(8 R / 2(\nu \tau)^{1 / 2}\right)-1 / 4\right]
$$

of such an isolate buoyant vortex ring structure is proportional to $\ln (\tau-1 / 2)$.

where

$$
\tau=\int_{0}^{t} R\left(t^{\prime}\right) d t^{\prime} / R(t),
$$

( $R$ is the radius of the ring) and differs significantly from the mean surge velocity curve obtained from observations. This suggest that the hydrodynamical effects alone cannot explain surge formation and dynamics. so that it is necessary to take into account some hydromagnetic effects, as well.

Some lab plasma experiments (see for example [7]) show that relatively stable plasma structures, called dynamically stable current filaments, can be formed. In the most general case these structures originate in toroidal vorticies and exibit proper longitudinal and azimuthal magnetic fields of approximately equal value. It is quite possible that in the magnetic flux tubes of the collimating external magnetic field, surge-like jets consist of similar MHD-structures which behave as plasmoids expelled by the action of the diamagnetic forces. In the electric field of the helix shaped cord of such MHD structures, electron acceleration is possible.

As for the braking force in the decelerated ascending phase, a simple explanation can be made if we take into account the fact that the effective size of the vortex core

$$
\delta=2(\nu \tau)^{1 / 2}
$$

where $V$ is the kinetic viscosity, grows rapidly with the height $z$. Besides, from the most general hydrodynamic consideration it can be shown [8] that in a moving fluid with a mean velocity $V$ of the velocity profile, pulsations will be generated when the pressure gradient $\mathrm{dP} / \mathrm{dy}$ transverse to $\mathbf{V}$ is proportional to $\mathrm{V}^{2}$. In [3] it was shown that the acting opposing force increases proportionally to the first or perhaps to the second power of the velocity of the surge.

If we assume that the surge-like jet plasma is moving in a magnetic flux tube (see the Figure), then the action of $\mathrm{dP} / \mathrm{dy}$ will lead to the broadening and dipping of the tube and to the appearance of $a$ small component $\mathbf{b}$ of the magnetic field, normal to $\mathbf{V}$. This disturbance 
of the magnetic tube will correspond to a local electric curent source. The generated Lorentz force $F=(1 / c) \mathbf{j} \times \mathbf{b}$ on the moving plasma will be directed in an opposite direction of $V$ and will act as a braking force.

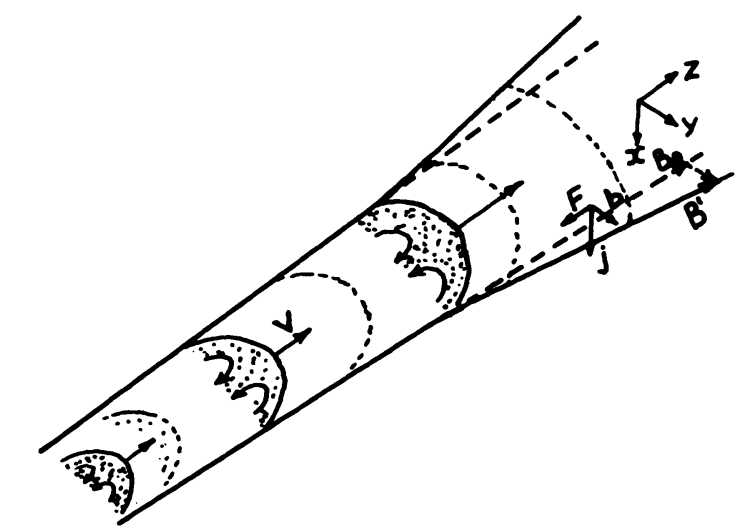

Figure. A sketch illustrating the action of the braking force on the surge plasma.

\section{REFERENCES}

1. Tandberg-Hanssen, E. (1974) Solar Prominences, D. Reidel Pbl. Co., Dordrecht.

2. Zirin, H. (1978) 'The ecology of prominences' in E. Jensen, P. Maltby and F. Q. Orrals (eds.), IAU Colloquium 44 'Physics of Solar Prominences', pp. 193-208.

3. Roy, J.-R. (1973) 'The dynamics of solar surges'. Solar Phys. 32, $139-151$.

4. Zachariadis, Th., Alissandrakis, C. E., Banos, G. (1985) Lecture Notes in Physics 233, Springer-Verlag, pp 304-306.

5. Dermendjiev, V. N., Buyukliev, G. T. (1987) 'Magnetic turbulent diffusion and the problem of solar surge formation' in L. Hejna and N. Sobotka (eds.) The Sun, Proc. of 10th European reg. astron. meeting of the IAU, Praha, pp. 113-116.

6. Turner, J. S. (1973) Buoyancy Effects in Fluids, Cambridge University Press.

7. Komelkov, V. S., Skvortsov, Y., Tereshenko, V. N., Zerevitinov, S. S. (1962) 'A dynamical current filament' in H. Maecker (ed.) Proc. of the Fifth Int. Conf. of Ionization Phenomena in Gases, vol. II, North-Holland Publ. Co., Amsterdam, pp. 2191-2200.

8. Lamb, H. (1945) Hydrodynamics. Dover Publications, New York. 